\title{
Reliable Communication Protocol for Wireless Sensor Networks by using Clustering and Cellular Learning Automata
}

\author{
Mojtaba Gorban Alizadeh \\ Computer, Islamic Azad University- \\ Ghareziaeddin Branch, Iran
}

\author{
Mahmood Javadi \\ Sama Technical and Vocational \\ Training College, Islamic Azad \\ University, Khoy Branch, Khoy, Iran
}

\author{
Ali Hosseinalipour \\ Computer, Islamic Azad University- \\ Ghareziaeddin Branch, Iran
}

\begin{abstract}
Limitation of energy consumption that impress lifetime of sensor network directly, is one of the major challenges in wireless sensor networks. In this paper, a confident communication protocol is presented that include low energy consumption for wireless sensor networks that dispense monotonous cargo of energy between sensors and increases network lifetime. This protocol is a cluster-Based one that originated from combination of DBS and clustering in wireless sensor networks by using cellular learning automata. Based on the proposed protocol, the cluster head nodes are identified in various phases based on parameters: 1) the amount of node energy 2) distance from the base station 3 ) the number of neighbors 4)the number of $\mathrm{CH}$ nodes in neighbors. Under this method, the percentage of reliability increase and energy consumption and communication delay between the sensors of wireless sensor network greatly reduced.
\end{abstract}

\section{Keywords}

Wireless sensor networks, clustering, learning automata, reduce energy consumption

\section{INTRODUCTION}

Wireless sensor networks that using for special environment stewardship and control, made of many cheap sensor nodes that dispersing densely in an environment. Data collected by sensors must be transmitted to a base station. In a direct transmittal, each sensor sends data to the center directly, that use more energy because of sensors great distance from the center. In opposite, patterns that shorten communication distances can do longer network life period and then multi-step communications are more economical and useful than one-step communications. But also in multi-step communications most of the nodes energy spending for communicating with other sensors, that result the sensors energy much consumption [3].

Grouping nodes in the cluster have been widely accepted by the research community until realize scalability; achieve higher energy efficiency and longer lifetime in WSN networks.

In Section 2 of this article, proposed clustering and cellular learning automata; In Section 3, examine two protocols that are base of the proposed method. In section 4, describe proposed protocol and Conclusions are made in Section 5.

\section{CLUSTERING AND CELLULAR LEARNING AUTOMATA}

\subsection{Clustering [5]}

Hierarchical routing protocols and data collection, predicate on a sensor cluster-based organizing that get composition and aggregation data, and lead to save remarkable energy. In hierarchical network structure, each cluster has a leader that called cluster head $(\mathrm{CH})$ and do special tasks generally (like data aggregation and composition). Also there are some normal nodes (SN) as the cluster members in each cluster.

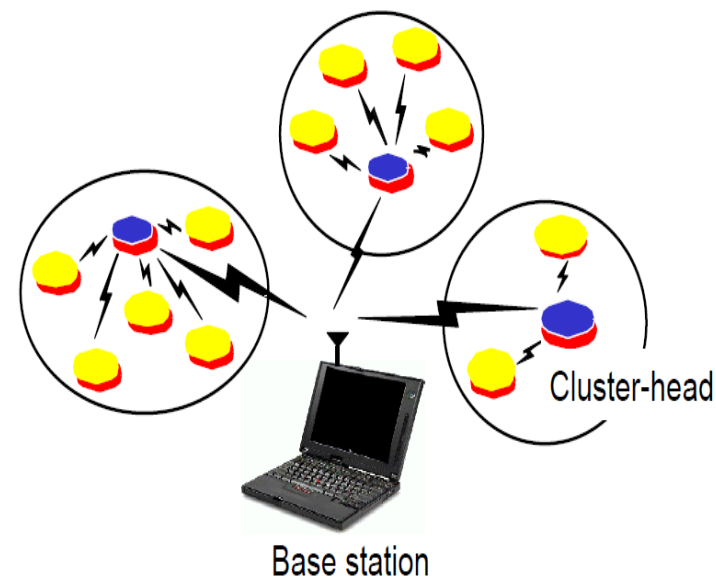

Figure 1: The hierarchical relationship between node and base stations and $\mathrm{CH}$

Cluster formation process, construct a two-level hierarchy that $\mathrm{CH}$ nodes constitute high level and (other) sensors that are cluster members constitute low level. Sensor nodes send data to their cluster head alternatively. $\mathrm{CH}$ nodes collect data (and thus reduced the number of retransmitting packets) and send them to the base station (BS). Transmittal to the BS can do directly or by inductor with other $\mathrm{CHs}$. Since the $\mathrm{CH}$ nodes at any time consign their data to longer distance (than normal nodes), therefore the rate of energy consumption in them is higher.

\subsection{Cellular learning automata [4]}

A learning automata is an abstract model that randomly select one action from own actions set and apply to environment or system. The Environment evaluates selected action by learning automata, and announced assessment results by a reinforcement signal to learning automata. Learning automata refresh own 
internal status with picked action and reinforcement signal, and then select next action. Figure 2 shows the relationship between learning automata and the environment [1-4].

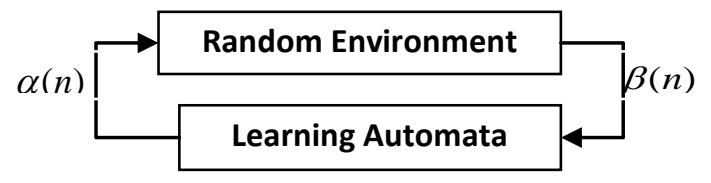

Figure 2: how communication Learning automata with environment

\subsection{Irregular Cellular Learning Automata (ICLA)}

An irregular cellular learning automata is a cellular learning automata (CLA) that is not limited in the rectangular grid at CLA. There are many applications that cannot be model with a rectangular grid, Such as wireless sensor networks, artificial immune systems, applications of graph and ... . An ICLA is defined as an undirected graph that each node of graph denotes a cell that is equipped to a learning automata. Learning automata in a certain cell, determined condition based on actions probability vector [1-4].

\section{RELATED WORKS}

\subsection{DBS protocol [1]}

To minimize the difference in consumption energy between the different nodes of the network And also reduce the number of waste transfers using earlier works, Distance-Based Segmentation (DBS) method offered that is a protocol based on clustering with comparative clusters that The distance between the nodes in the cluster policy is compiled from a base station $[5,6,7,10]$. This protocol based on distance from the base station does justly distribution of $\mathrm{CH}$ nodes in the network. The basic idea of this protocol is nodes that have further distance from the base station than nearer nodes should be lesser selected as cluster head $(\mathrm{CH})$ until be sure not engender many difference between energy level of a near node and a far node. In DBS protocol, cluster form process is same the LEACH protocol but $\mathrm{CH}$ selection method and MAC protocol are different, So that provide effective communication in terms of energy consumption, reliability and network delay. DBS divide network considering the concentric circles around the base station to even area loops and burden different number of cluster according to loop distance to base station for each loop. In near loops, possibility of become $\mathrm{CH}$ rather than far loop; thereupon number of $\mathrm{CH}$ at near loop will be further $[5,6,7,10]$.

\subsection{Clustering in wireless sensor networks by using cellular learning automata [2]}

In this method it is assumed that all nodes in sensor networks are the same and each network node has two modes can be:

\section{Cluster head node $(\mathrm{CH})$}

Normal node $(\mathrm{CN})$

The nodes that place in cluster head situation, is responsible for information receipt from other nodes in the network and send them to the Sink node. Measure energy consumption is higher than normal in cluster head. In normal node state, collect production data in own sensor radius Node, and send to the cluster head node. In this mode, nodes consume less energy than the $\mathrm{CH}$ mode. The main criteria that are considered include:

\section{1) The amount of use energy \\ 2) Connection to the network}

Algorithm contain two phase: clustering phase and steady state phase, that in clustering phase by using cellular learning automata technique sensor nodes select one of the cluster head or normal state, and form clusters and each normal type recognize own cluster head. Then start steady state phase that in this phase, normal nodes with timing program that cluster head appoint for them, send collected data to the cluster head and also cluster head send information to the sink node. Meantime, in the steady state phase, whenever cluster head node energy comes lower from a certain amount, cluster head node selected one node with higher energy as cluster head $[8,9]$.

\section{THE PROPOSED PROTOCOL}

Basis of the proposed protocol is the DBS protocol. All formation process of clusters and MAC protocols is same the DBS. (In DBS protocol type of a MAC protocol, CSMA / CA is called MACA is used that in addition to eliminating the problem of hidden stations, reduces significant numbers of collisions between the packages, also reduce excessive delays in the network at package arriving to the base station.) Just another step has been added to the $\mathrm{CH}$ selection stage. In addition to select $\mathrm{CH}$ based on distance of base station, with cellular learning automata, nodes that are $\mathrm{CH}$ in each stage, their status become steady and not enter into next learning stage. In other words, each node with using the DBS protocol has conditions to be $\mathrm{CH}$, select and take privilege. Then each node, will inform their neighbors to the adjacent nodes. Apiece have further number of neighbors and lesser privilege than other, select own as $\mathrm{CH}$ and inform to the neighbors. A node that selected as $\mathrm{CH}$ or was in the neighborhood of the $\mathrm{CH}$ nodes, its condition to be stable and not be entering to next learning stage. But Nodes that their status still unclear, determine status using the technique of irregular cellular learning automata (ICLA). With each select, node being $\mathrm{CH}$ measure based on various parameters decreased or increased: 1) amount of node energy 2) distance from the base station 3) the number of neighbors 4) the number of neighbor $\mathrm{CH}$ nodes. By this way, efficiency of the DBS protocol in energy consumption and lifetime of the network will be multiplied. Indeed, the proposed protocol is combination of DBS protocol and clustering method in wireless sensor networks with using cellular learning automata.

The proposed protocol has two phases: 1) start-up phase (clustering) and 2) steady state phase. Different phase of proposed method is described below.

\section{- Start-up phase}

Since the network that using DBS protocol is equivalent with network that in each loop using LEACH protocol, the $\mathrm{CH}$ selection in LEACH is scan:

In LEACH Protocol, based on probability function (1) $\mathrm{CH}$ is selected. The $\mathrm{CH}$ selection is so that each sensor selects a random number between zero and one randomly. If this number is smaller than specified threshold in the below relation, the node is selecting as $\mathrm{CH}$ :

$$
P_{i}(t)= \begin{cases}\frac{P}{1-p *\left(r \bmod \frac{1}{p}\right)} & \text { if } C_{i}(t)=1 \\ 0 & \text { if } C_{i}(t)=0\end{cases}
$$


In the above relation, $\mathrm{p}$ is proposed percent to network for the number of $\mathrm{CH}$ that is predetermined.

In start-up phase Clusters should be formed and characterized $\mathrm{CH}$ nodes. Appoint of $\mathrm{CH}$ nodes in the proposed protocol, is performed in two stages. Stage II consist three parts.

In first stage, with this supposition that $\mathrm{N}$ number of nodes pervade monotonously in network area; averagely $\mathrm{N} / \mathrm{m}$ number of nodes will have in each loop that $\mathrm{m}$ is loop number demonstrator; innermost loop has least index (index 1); in loop $\mathrm{j}$, node $\mathrm{i}$ with below probability in cycle $\mathrm{r}$ that started in time $\mathrm{t}$, in first stage selected: $k_{\text {segment }}{ }^{j}$

$$
\begin{aligned}
& P_{i, j}(t)=\left\{\begin{array}{c}
\frac{k_{\text {segment }^{j}}}{\frac{N}{m}-k_{\text {segment }^{j}} *\left(r \bmod \frac{N / m}{k_{\text {segment }}{ }^{j}}\right)} \\
0 \quad \text { if } C_{i, j}(t)=0
\end{array}\right. \\
& =\left\{\begin{array}{cc}
\frac{p_{\text {segment }}{ }^{j}}{1-p_{\text {segment }^{j}} *\left(r \bmod \frac{1}{p_{\text {segment }}{ }^{j}}\right)} & \text { if } C_{i, j}(t)=1 \\
0 & \text { if } C_{i, j}(t)=0
\end{array}\right.
\end{aligned}
$$

In this relation, indicator function $\mathrm{C}_{\mathrm{i}, \mathrm{j}}(\mathrm{t})$ is equal one while node $\mathrm{i}$ in loop $\mathrm{j}$ has selection sufficiency in time t. (namely in ( $\mathrm{r}$ mod $\left.\left(1 / p_{\text {segment }}\right)\right)$ past around not be $\left.\mathrm{CH}\right)$, in otherwise is zero. If loop number is odd, $\mathrm{p}_{\text {segment } \mathrm{j}}$ in below form calculate:

$$
\text { Psegment }_{j}=\left\{\begin{array}{c}
P_{L E A C H} \quad \text { if } j=\frac{m+1}{2} \\
P_{L E A C H}+\left(\frac{m+1}{2}-j\right) * \partial_{p} \quad \text { otherwise }
\end{array}\right.
$$

$\mathrm{P}_{\text {LEACH }}$ is probability be $\mathrm{CH}$ in LEACH and is equal to $\mathrm{k}_{\text {opt }} /$ $\mathrm{N}$ that $\mathrm{k}_{\mathrm{opt}}$ is the optimum number of clusters $(\mathrm{CH})$ in LEACH and $\partial_{p}$ is difference between likely be $\mathrm{CH}$ in two neighbor loops. Mostly start with a small amount of $\partial_{p}$ and it will increase gradually to reach an optimum value. If $\partial_{p}$ be greater than this optimum value, it will reduce network performance in terms of energy consumption, Because in far clusters about any cluster head not selected and with increment of cluster size (as regards the direct relationship between $\partial_{p}$ and the size of the cluster is established), their members have forced to send data to long distances to reach the $\mathrm{CH}$ and thereupon their energy will spend soon. In the state that loop number for network be even, the similar relation with relation (3) will be use that ensure loops probability dispensation environs of $\mathrm{P}_{\text {LEACH. }}$.

$\mathrm{p}_{\text {segment } \mathrm{j}}$ as the initial point for the sensors is considered. In fact, the proposed protocol in the first stage in the DBS phase, with specified $\mathrm{p}_{\text {segment } \mathrm{j}}$ as the initial point for the sensors, entered to the second stage, the clustering fhase in wireless sensor networks by cellular learning automata. (The default is the node that had more point; there is less chance for being $\mathrm{CH}$ ).

In stage two, the first part, with earned point in the first stage, each node informed number of neighbors in a message to the contiguous nodes. Then the node that has most number of neighbors than own contiguous nodes and its point is less than other nodes, select own as $\mathrm{CH}$ and inform to the neighbor nodes. In this part, nodes that be $\mathrm{CH}$ or are in the neighbor of a $\mathrm{CH}$ node, not entered in the next learning stage and their situation be steady.

In the second part of stage II, the nodes that their status are not known in before part, determine it by using the irregular cellular learning automata (ICLA) technique. For use from this technique, correspond sensor network, spot one irregular cellular learning automata that each node in network not reach to steady state in the before part, is equivalent with a cell in ICLA. In ICLA, two cells are neighbor if the distance of corresponding nodes is less than communication range. Corresponding automata of each node can select one of the two non- $\mathrm{CH}$ and $\mathrm{CH}$ alphabet based on the probability vector. In each cycle Automata of each node based on the probability related to each action, choose one randomly. With each selection, the probability of nodes that be cluster head based on different parameters can be increased or decreased. The parameters for clustering are considered are: 1) the energy level of nodes. 2) The number of nodes neighbor. 3) The number of neighboring $\mathrm{CH}$ nodes. Generally, these parameters can be divided into two general categories: Parameters related to energy, which tries to select nodes with more energy as cluster head and parameters relating to the manner and quality of clustering infrastructure, which tries to form the proper number of cluster and develop balanced clusters. For this reason that can associate above two sect of parameters at ideal measure in cluster production, spot two coefficients as their summation is equal to one.

With notice to the parameters listed above, with selection any act by nodes automata altered possibility of done. For Rewarding or fining picked act, the below relations are used that (a) is reward coefficient and (b) is fine coefficient.

$P_{i}(n+1)=P_{i}(n)+a\left[1-P_{i}(n)\right]$
$P_{j}(n+1)=1-P_{i}(n+1)$

$P_{i}(n+1)=(1-b) P_{i}(n)$

$P_{j}(n+1)=1-P_{i}(n-1)$

In each cycle every node, after selecting an action, based on relations (6) and (7), $\beta_{\mathrm{i}}$ strength signal can be calculate. If $\beta$ equal to one, the selection act by using relation (5) will be penalty, and if $\beta_{\mathrm{i}}$ equal to zero, by using relation (4) will be rewarded.

If selected act is that node be $\mathrm{CH}\left(\alpha_{i}(n)=1\right)$

,$\beta_{\mathrm{i}}(\mathrm{n})$ value obtained by relation (6):

$$
\beta \mathrm{i}(\mathrm{n})=\left\{\begin{array}{cc}
0: \mathrm{W}_{\theta} \Psi_{i}(n)+\mathrm{W}_{u}\left(\frac{\lambda_{i}}{2}+\frac{\tau_{i}(n)}{2}\right)>0 \\
1: \quad \text { otherwise }
\end{array}\right.
$$

So that

$$
\lambda_{i}=\frac{\left|N_{i}-N_{\mu}\right|}{N_{i}-N_{\mu}} \quad \psi_{i}(n)=\frac{\left|e_{i}(n)-e_{\mu}(n)\right|}{e_{i}(n)-e_{\mu}(n)}
$$

$e_{i}(n)$ is residual energy of node in cycle n. $e_{\mu}(n)$ is average energy of node's neighbor. $\mathrm{N}_{\mathrm{i}}$ is Number of node's neighbor and $N_{\mu}$ is average number of neighbors of nodes that are neighbor to node.

$$
\tau_{i}(n)=\left\{\begin{array}{lll}
-1 & : & \sum_{j=0}^{N i} \alpha_{j}(n)>0 \\
1 & : & \text { otherwise }
\end{array}\right.
$$

$\alpha_{j}(n)$ is node j's selected act.

The constant coefficient $\mathrm{w}_{\mathrm{e}}$ is appropriate burden to the energy parameter in clustering algorithm and the constant coefficient $\mathrm{w}_{\mathrm{u}}$ is appropriate burden to the parameters relevant to quality of clustering infrastructure. These two factors are numbers between zero and one as the sum of these coefficients is equal to one. 
If selected act is the selection of node as a non- $\mathrm{CH}$ node $\left(\alpha_{i}(\mathrm{n})=0\right) \beta_{\mathrm{i}}(\mathrm{n})$ value obtained by relation (7):

$$
\beta \mathrm{i}(\mathrm{n})=\left\{\begin{array}{c}
1: \mathrm{W}_{\theta} \Psi_{i}(n)+\mathrm{W}_{u}\left(\frac{\lambda}{2}+\frac{\tau_{i}(n)}{2}\right)>0 \\
0:(7)
\end{array}\right.
$$

This part do in several cycle, in each cycle every sensor's node select one of be cluster head or not statues based on probability vector and send a message to all neighbors as diffused that message contain node's selected act, measure of remainder energy and number of neighbors. After distinctive time period that all nodes received neighbor's messages, every node based on relation (4), (5) give reward or penalty to selected act and actually probability of node be cluster head increase or decrease and in the next cycle nodes based on the new probability select a new mode and operations is repeated.

Every node that it probability of be cluster head reach to near one or zero, based on probability vector select a state and reach to steady situation. This algorithm continues until a certain percentage of nodes (95\% in done trials) reach to steady condition. At this time, all nodes select their situations and this stage ends.

In part three of second stage, after clarifying the status of the nodes and determining cluster heads in the two previous parts, clusters are formed. At this stage, cluster head nodes send messages containing location of cluster head nodes to their neighbor nodes as diffused message. Then normal nodes select nearest node among neighbors of cluster head as cluster head. For creation cluster, every normal node send (JOIN_PACKET) a package to cluster head, and cluster head nodes with collection these packages recognize cluster members and cluster are formed, then create a schedule and send a schedule package (SCH_PACKET) to cluster members to inform sending time of data of any cluster members to them.

\section{- Steady state phase}

After clustering phase and creating cluster, start steady state phase. In this phase normal nodes send data to cluster head nodes alternatively and with distinctive spaces of time, and cluster head nodes send data to sink. For interception from intervention in data sending, nodes member of cluster use a schedule program for sending data. (As previously mentioned, proposed protocol use from MACA protocol that main idea in this method is which sender provoke receiver to send a short package for own environs station until nodes that are in receiver range and hear this short package, refusal from sending information in between receiving major package time.) Head cluster nodes consume energy rather than other nodes and waste quickly and network stop. To avoid this problem, this paper use from changing head cluster nodes in a cluster. That is, whenever remainder energy of head cluster node is lesser than amount of energy of cluster nodes and of a lower threshold, being head cluster assigned to a node that has the highest energy.

\section{SIMULATION AND CONCLUSION}

It is simulated a wireless sensor network in an area the size of $100 * 100$ square meters and with a uniform distribution of different sensors, the first time 100 sensors and the second 200 sensor and the third 300 sensor and the fourth 400 sensor and for the fifth time 500 sensor randomly by MATLAB software. In this simulation, all nodes and sink node with specific characteristics are in the area. In this paper have considered 0.5 Joules for the initial energy of sensors and 1000 cycle for running time of simulation and consumption energy is calculated based on Table 1 .
Table 1: RF parameters used in the simulation

\begin{tabular}{|c|c|}
\hline Function & Consumption energy \\
\hline Send and receive data & Eelec $=27 \mathrm{~nJ} / \mathrm{bit}$ \\
\hline Data Aggregation & $\mathrm{EDA}=5 \mathrm{~nJ} / \mathrm{bit} / \mathrm{signal}$ \\
\hline The booster ifdmaxtoBS $\leq \mathrm{d} 0$ & $\epsilon f_{\mathrm{S}}=10 \mathrm{pJ} / \mathrm{bit} / \mathrm{m} 2$ \\
\hline The booster ifdmaxtoBS $\geq \mathrm{d} 0$ & $\epsilon \mathrm{mp}=0.0013 \mathrm{pJ} / \mathrm{bit} / \mathrm{m} 4$ \\
\hline
\end{tabular}

In this paper took two tests of different aspects of network, at first time have eclipsed the energy of cluster heads than the average energy of normal nodes. The simulation results shown in Figure 3 indicates that in the proposed method the rate of cluster head energy compared with other methods retains more energy and as a result, compared with the DBS algorithms, clustering with learning automata and LEACH increases the network lifetime and High -lived cluster heads can increase the reliability of data sent and received.

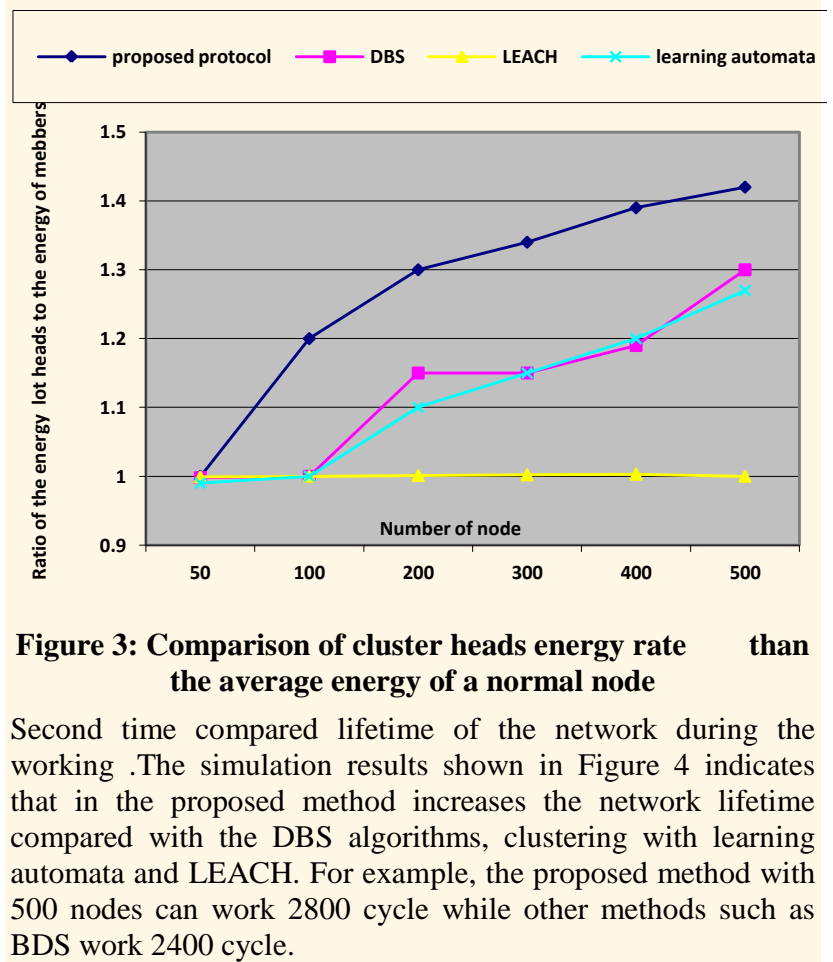




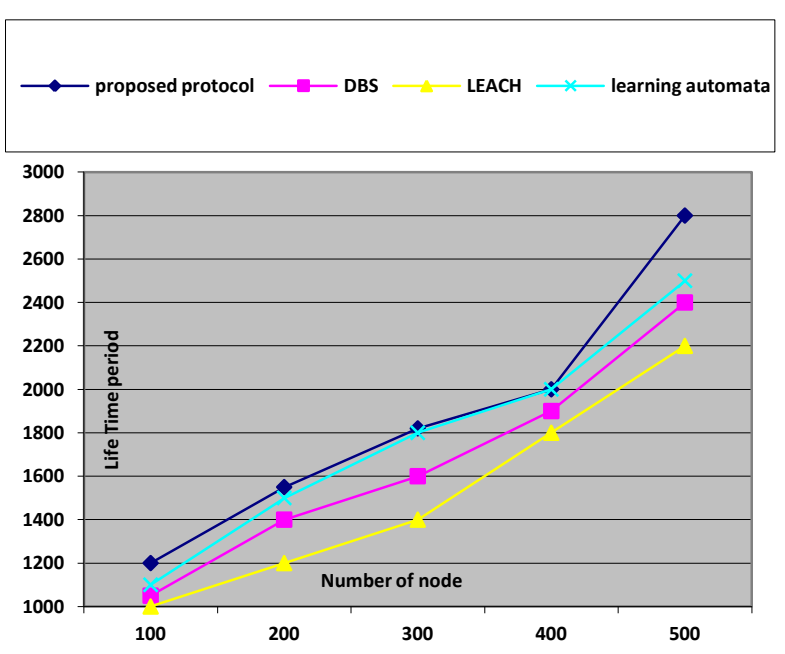

Figure 4: Comparison of network lifetime with different number of nodes in the network, working in different periods

In this paper, a confident communication protocol for wireless sensor networks is introduced based on the combination of DBS protocol and clustering method in wireless sensor networks using cellular learning automata. the results show that the proposed protocol have improved DBS method for selecting appropriate cluster heads using cellular learning techniques have improved, Is One of the main criteria is the quality of service in wireless sensor networks, increases the $\mathrm{CH}$ near the base station to distribute more evenly .

\section{REFERENCES}

[1] M. R. Meybodi, H. Beigy, "New Class of Learning Automata Based Scheme for Adaptation of Back propagation Algorithm Parameters", Proc. Of EUFIT-98, Sep. 7-10, Achen, Germany, pp. 339-344, 1998.

[2] B. J. Oommen, D. C. Y. Ma, "Deterministic Learning Automata Solution to the Keyboard Optimization Problem", IEEE Trans. On Computers, Vol. 37, No. 1, pp. 2-3, 1988.
[3] H. Beigy, M. R. Meybodi,"Optimization of Topology of Neural Networks Using Learning Automata", Proc. Of 3th Annual Int. Computer Society of Iran Computer Conf. CSICC-98, Tehran, Iran, pp. 417-428, 1999.

[4] A. A. Hashim, S. Amir, p. Mars, "Application of Learning Automata to Data Compression, In Adaptive and Learning Systems", K. S. Narendra (Ed), New York: Plenum Press, pp. 229-234, 1986.

[5] Heinzelman W. R., A. P. Chandrakasan and H Balakrishnan, "Energy-Efficient Communication Protocol for Wireless Microsensor Networks", Proc. of the 33rd IEEE International Conference on System Sciences, Honolulu, USA, Jan. 2000,pp. 1-10.

[6] Handy M. J., M. Haase and D. Timmermann, "Low Energy Adaptive Clustering Hierarchy with Deterministic Cluster-Head Selection", Proc. of 4th IEEE International Conference on Mobile and Wireless Communications Networks, Stockholm, Sweden, 2002, pp. 368-372.

[7] Heinzelman W. R., A. P. Chandrakasan and H. Balakrishnan, "An Application-Specific Protocol Architecture for Wireless Microsensor Networks", IEEE Transactions on Wireless Communications, vol. 1, no. 4, Oct. 2002, pp. 60-670.

[8] Farajzadeh, N. and Meybodi, M. R., "Learning Automatabased Clustering Algorithm for Sensor Networks", Proceedings of 12 th Annual CSI Computer Conference of Iran, Shahid Beheshti University, Tehran, Iran, pp.780-787 , Feb.20-22,2007.

[9] Esnaashari, M. and Meybodi, M. R., "A Cellular Learning Automata based Clustering Algorithm for Wireless Sensor Networks", Sensor Letters, 2008.

[10] N.Aminit.Fazeli, S.G.Miremadi, M.T.Manzuri, "DistanceBased Segmentation: An Energy-Efficient Clustering Hierarchy for Wireless Microsensor Networks", IEEE Communication Networks and Services Research, 2007. CNSR '07. Fifth Annual Conference. 